\title{
Analysis of FK506-mediated functional recovery and neuroprotection in a rat model of spinal cord injury indicates that EGF is modulated in astrocytes
}

\author{
$\mathrm{JUN} \mathrm{CAI}^{1,2^{*}}, \mathrm{YU} \mathrm{SUN}^{3 *}, \mathrm{ZAOYANG} \mathrm{YIN}^{4}, \mathrm{DAODE} \mathrm{WANG}^{4}, \mathrm{KUN} \mathrm{SHI}^{4}$, \\ YUXUAN FU ${ }^{2}$, XIAOJIAN $\mathrm{CAO}^{4}$ and YINGBIN $\mathrm{GE}^{2}$ \\ ${ }^{1}$ Department of Orthopedics, Clinical Medical College of Yangzhou University, Yangzhou, Jiangsu 225001; \\ ${ }^{2}$ Department of Physiology, The First Affiliated Hospital of Nanjing Medical University, Nanjing, Jiangsu 210029; \\ ${ }^{3}$ Department of Orthopedics, Subei People's Hospital, Yangzhou, Jiangsu 225001; \\ ${ }^{4}$ Department of Orthopedics, The First Affiliated Hospital of Nanjing Medical University, Nanjing, Jiangsu 210029, P.R. China
}

Received November 10, 2015; Accepted December 9, 2016

DOI: $10.3892 /$ etm.2018.6283

\begin{abstract}
The present study aimed to evaluate whether the application of tacrolimus (FK506) could improve functional recovery in spinal cord injury $(\mathrm{SCI})$ rat models by activating astrocytes, and to further investigate the underlying mechanisms of this action. Male Sprague-Dawley rats $(n=56)$ were used to establish moderate SCI models, which were induced at the T10 spinal segment by dropping a 10-g weight from a height of $25 \mathrm{~mm}$ using a New York University Impactor device. The rats were randomly separated into the FK506 or control group ( $\mathrm{n}=28$ per group). Rats were treated with FK506 $(0.5 \mathrm{mg} / \mathrm{kg})$ or saline intravenously $30 \mathrm{~min}$ after sustaining the injury. Functional recovery was evaluated over 42 days following the injury, and epidermal growth factor (EGF) levels were detected. The astrocytes were treated with FK506 in vitro, and the EGF mRNA and protein expression levels were analyzed using reverse transcription-quantitative polymerase chain reaction and ELISA, respectively. DNA microarray analysis was also performed to evaluate the genes in astrocytes. Rats in the FK506 group had improved locomotor functional recovery compared with those of control group. Furthermore, FK506 upregulated EGF expression of astrocytes both in vivo and
\end{abstract}

Correspondence to: Dr Xiaojian Cao, Department of Orthopedics, The First Affiliated Hospital of Nanjing Medical University, 300 Guangzhou Road, Nanjing, Jiangsu 210029, P.R. China

E-mail: xiaojiancao@gmail.com

Dr Yingbin Ge, Department of Physiology, The First Affiliated Hospital of Nanjing Medical University, 300 Guangzhou Road, Nanjing, Jiangsu 210029, P.R. China

E-mail: ybge@163.com

*Contributed equally

Key words: spinal cord injury, FK506, astrocytes, functional recovery, neurite outgrowth in vitro. Subsequent to treatment with FK506-conditioned medium (CM), the length of neuronal cells increased $61.06 \%$ on the first day, and increased $56.4 \%$ on the third day compared with those of C-CM group. Furthermore, addition of anti-EGF neutralizing antibodies could interrupt the promotion of neurite outgrowth by FK506-CM. The present study indicates that astrocytes have an important role as mediators of FK506-improved spinal cord function recovery, and this partially clarifies the role of cell-cell interaction through modulating EGF in this process.

\section{Introduction}

Spinal cord injury (SCI) is a traumatic event, often resulting in permanent motor and sensory deficits such as paraplegia and tetraplegia (1). It has been estimated that between 250,000 and 500,000 people sustain a SCI each year worldwide (2). This debilitating condition results in significant physical and emotional burden to a substantial number of affected individuals, and also imposes high economic costs to healthcare systems (3). As the consequences of SCI are severe, intense research to elucidate the underlying pathophysiological mechanisms and to discover potential therapeutic strategies are in demand.

The biological processes resulting in traumatic SCI may be categorized into primary and secondary injury, followed by regeneration and functional recovery (4). Typically, the primary injury of the spinal cord resulting from contusion or compression is a local, segmental-limited damage, and the secondary expansive phase results in further destruction of neuronal and glial cells, in addition to invasive degeneration of the surrounding spinal cord tissue (5). The repair capacity of the central nervous system is limited due to decreased intrinsic growth capacity and a non-permissive environment for axonal elongation, while regenerative processes are hindered by different factors, such as inhibitory growth factors and the glial scar at the site of the lesion (1). Recent neuroscience advances have facilitated the prevention and cure for the debilitating effects of SCI, and neuroprotection/neuroregeneration 
approaches to promote axonal sprouting is a promising form of therapy (2). An increasing body of evidence has suggested that the predominant glial cell type reactive astrocytes can provide essential activities that protect tissue and preserve function after SCI (3). In addition, Davies et al (4) found that transplantation of specific human astrocytes could promote functional recovery after SCI. However, the role of astrocytes has yet to be fully elucidated.

Tacrolimus (FK506), an FDA-approved immunosuppressive agent, is widely used to prevent the acute rejection of allograft transplants after transplantation (5). Recently, FK506 has been reported to exhibit both neuroprotective and neuroregenerative properties for the treatment of peripheral nerve injuries (6). Furthermore, FK506 is able to enhance axonal regeneration and improve functional recovery in animal models after SCI (7). However, the definite mechanisms of FK506 in terms of its neuroprotective and neuroregenerative action have yet to be elucidated.

Wildering et al (8) previously demonstrated that an epidermal growth factor (EGF) homolog could promote axonal regeneration, substantiating existing molecular evidence that has suggested that the EGF family is involved in peripheral nerve regeneration. Astrocytes produce a large array of neurotrophic factors, including EGF (9). Thus, the aim of the present study was to assess whether FK506 is able to enhance axonal regeneration and improve functional recovery by activating astrocytes, and to further investigate the possible mechanisms of action. For this purpose, a rat model of SCI was established. Functional recovery, EGF expression levels and the length of neuronal cells were assessed following the treatment of rats with FK506.

\section{Materials and methods}

Animals grouping and FK506 preparation. Male Sprague-Dawley rats ( $\mathrm{n}=56$; age, 10 weeks; weight, 280-320 g) were purchased from BetterBiotechnology Co., Ltd. (Nanjing, China) and used in the present study. The animals were housed under a 12-h dark/light cycle at a temperature between 23 and $28^{\circ} \mathrm{C}$, and had free access to food pellets and water. All animal experiments were approved by the Animal Care and Research Committee of the Nanjing Medical University (Nanjing, China). The rats were randomly divided into the K506 treatment or control group ( $\mathrm{n}=28$ per group) by simple random sampling without replacement approach. The rats in each group were then separated into different subgroups depending on the day of sacrifice with a lethal dose of sodium pentobarbital (days 1 , 3, 7 and 14; four rats per day; $100 \mathrm{mg} / \mathrm{kg}$; Shanghai Chemical Reagent Co., Ltd., Shanghai, China) followed by cardiac perfusion with heparinized saline and $4 \%$ paraformaldehyde, and used for functional recovery evaluation $(n=12)$.

A total of 5 mg FK506 (Sigma-Aldrich; Merck Millipore, Darmstadt, Germany) was dissolved in $500 \mu \mathrm{l}$ dimethyl sulfoxide (DMSO; Sigma-Aldrich) and stored at $-20^{\circ} \mathrm{C}$ until use. The final concentration of DMSO was maintained at $\leq 0.1 \%$.

Surgical procedures. Acute SCI was induced as previously reported using the New York University (NYU) weight-drop device (10). Briefly, after anesthesia by intraperitoneal administration of $10 \%$ chloral hydrate $(400 \mathrm{mg} / \mathrm{kg}$; Sigma-Aldrich), the spinal cords at the T10 level were exposed after laminectomy and subjected to a weight-drop impact of a 10-g rod falling from a height of $25 \mathrm{~mm}$ with the NYU impactor (11) to produce a moderate SCI model. Next, the muscles and skin were sewn in layers. After $30 \mathrm{~min}, 0.5 \mathrm{mg} / \mathrm{kg}$ FK506 was administered intravenously to the rats. Rats in the control group were administered the equivalent dose of normal saline. In addition, the bladders of these rats were emptied manually twice a day.

Functional recovery evaluation. The open-field locomotion test was used to evaluate the functional recovery after SCI. It was observed by two blinded independent investigators and scored using the standardized Basso, Beattie and Bresnahan (BBB) locomotor scoring system (12). BBB scores range between 0 (flaccid paralysis) and 21 (normal gait). Prior to testing, rats were acclimatized to the testing environment (90-cm diameter plastic wading pool; $4 \mathrm{~cm}$ in height). The test was performed prior to surgery and on days $3,7,14,21,28$, 35 and 42 post-operation. BBB scores were averaged for each group by both examiners.

Immunohistochemical analysis. The rats were sacrificed on days 1, 3, 7 and 14 after injury, followed by cardiac perfusion with heparinized saline and $4 \%$ paraformaldehyde in $0.1 \mathrm{M}$ phosphate buffer. Subsequent to perfusion, the T10 region of the spinal cord was removed and fixed in $4 \%$ paraformaldehyde overnight at $4^{\circ} \mathrm{C}$. Then, the tissues were cryoprotected with $20 \%$ and then $30 \%$ sucrose in phosphate-buffered saline (PBS). Tissues were frozen on dry ice and cryosectioned at $8 \mu \mathrm{m}$ using a microtome cryostat (Leica model CM1850; Leica Microsystems, Inc., Buffalo Grove, IL, USA), and the $8 \mu \mathrm{m}$ sections were collected on Superfrost Plus glass slides. For the immunohistochemical reactions, sections were rehydrated in 0.1 M PBS, permeabilized with $0.2 \%$ Triton X-100 for $5 \mathrm{~min}$, washed twice with PBS, blocked with $5 \%$ bovine serum albumin (BSA) in PBS for $30 \mathrm{~min}$ at room temperature, and subsequently incubated with the monoclonal antibody against glial fibrillary acidic protein (GFAP; anti-GFAP-mouse-IgG; cat. no. sc-65343) and anti-EGF-rabbit-IgG (cat. no. sc-03; both 1:100; Santa Cruz Biotechnology, Inc., Dallas, TX, USA) overnight at $4^{\circ} \mathrm{C}$. After washing 3 times for $5 \mathrm{~min}$ in PBS, the samples were incubated with anti-mouse-IgG-Alexa 488 and anti-rabbit-IgG-Alexa 568 (both 1:500; Santa Cruz Biotechnology, Inc.) dissolved in PBS for $1 \mathrm{~h}$ in the dark at $24^{\circ} \mathrm{C}$. Finally, the nuclei were stained with DAPI $(5 \mathrm{mg} / \mathrm{ml}$; Invitrogen; Thermo Fisher Scientific, Inc., Waltham, MA, USA) for $5 \mathrm{~min}$ at room temperature. The slides were observed under a Zeiss Axio ImagerA1 fluorescence microscopy (Carl Zeiss AG, Oberkochen, Germany) with an attached color camera (Evolution $^{\mathrm{TM}}$ MP; Media Cybernetics, Inc., Rockville, MD, USA). The control slides consisted of omitting the incubation with the primary antibody, and no reactivity was observed.

Astrocytes cell culture. Primary cultures of rat spinal cord astrocytes were prepared from two-day old Sprague-Dawley rats as previously described, with modifications (13). Briefly, the meninges were carefully removed and spinal cords were dissected under sterile conditions. Spinal cords were dissociated in $0.25 \%$ trypsin for $5 \mathrm{~min}$ at $37^{\circ} \mathrm{C}$, and the digestion 
was terminated with $1 \mathrm{ml}$ fetal bovine serum (FBS). Then, the cell suspension was centrifuged at $200 \mathrm{x} \mathrm{g}$ for $5 \mathrm{~min}$ at room temperature. The cells were cultured in 30-mm Petri dishes at $37^{\circ} \mathrm{C}$ in a $5 \% \mathrm{CO}_{2}$ humidified atmosphere. After the conventional trypsinization procedure, cells were seeded into 60-mm Petri dishes and cultured until they reached confluence. Prior to experiments, the purity of astrocytes was $>95 \%$, as determined by immunocytochemistry with the astrocytic marker GFAP.

FK506 treatment and conditioned medium preparation. After reaching 90-95\% confluence, astrocyte monolayers were washed with PBS, incubated with serum-free Dulbecco's modified Eagle's medium (DMEM; Gibco; Thermo Fisher Scientific, Inc.) for $24 \mathrm{~h}$ to allow cells to reach a non-dividing G0-phase in the cell cycle (14), then treated with or without $20 \mu \mathrm{M}$ FK506 (Sigma-Aldrich) in serum-free DMEM for $24 \mathrm{~h}$. The conditioned media (CM) of the control group (C-CM) and FK506-treated group (FK506-CM) were collected, centrifuged at 7,500 x g for $20 \mathrm{~min}$ by an Amicon Ultra-4 3K centrifugal filter device (Merck Millipore) to remove residual FK506, then diluted to the initial volume with neurobasal medium (Gibco; Thermo Fisher Scientific, Inc., Grand Island, NY, USA). Subsequently, the conditioned media were stored at $-80^{\circ} \mathrm{C}$ and used within one week.

Reverse transcription-quantitative polymerase chain reaction (RT-qPCR) analysis. At $8 \mathrm{~h}$ after the administration of FK506, total RNA was extracted from astrocytes using TRIzol (Invitrogen; Thermo Fisher Scientific, Inc.) in accordance with the manufacturer's protocol. Single-strand cDNA was synthesized from the total RNA using the Superscript One-Step RT-PCR system (Invitrogen; Thermo Fisher Scientific, Inc.). The obtained cDNA was used for RT-qPCR. RT-qPCR reactions were performed using an ABI Prism 7000 sequence detection system (Applied Biosystems; Thermo Fisher Scientific, Inc., Foster City, CA, USA) with the GoTaq qPCR Master Mix (Promega Corporation, Madison, WI, USA). Primer sequences were as follows: EGF, forward, 5'-CTTAGG GATGTGGGGGACTT-3' and reverse, 5'-TTGGGCTGTTGG TGTTCCTC-3' for EGF; GAPDH forward, 5'-TGAACGGGA AGCTCACTGG-3' and reverse, 5'-TCCACCACCCTGTTG CTGTA-3'. The qPCR cycling conditions were as follows: Initial denaturation at $95^{\circ} \mathrm{C}$ for $10 \mathrm{~min}$, followed by 45 cycles of $15 \mathrm{sec}$ denaturation at $95^{\circ} \mathrm{C}$, annealing for $10 \mathrm{sec}$ at $58-60^{\circ} \mathrm{C}$, $20 \mathrm{sec}$ extension at $72^{\circ} \mathrm{C}$ and a final dissociation phase at $60-95^{\circ} \mathrm{C}$. Relative mRNA levels of all genes were normalized against the levels of GAPDH using the $\Delta \Delta \mathrm{Cq}$ method (15). The experiments were repeated three times.

DNA microarray analysis. Subsequent to treatment with $20 \mu \mathrm{M}$ FK506 for $8 \mathrm{~h}$, astrocytes cells were charged and loaded into TRIzol at a density of $1 \times 10^{6}$ cells $/ \mathrm{ml}$. Shanghai Kangcheng Biological Co., Ltd. (Shanghai, China) completed the follow-up experiments of gene microarray analysis (16). In brief, RNA was reverse-transcribed to cDNA, labeled with Cy3 dye, and then subjected to one-color hybridization (17). Following hybridization and washing, the slides were scanned using the Agilent DNA Microarray scanner G2505B (Agilent Technologies, Inc., Santa Clara, CA, USA). The resulting text files extracted by Agilent Feature Extraction Software (version 9.5.3) were introduced into Agilent GeneSpring GX software (version 11.0) for further analysis. The microarray datasets were normalized and differentially expressed genes were identified through a fold change analysis.

Enzyme-linked immunosorbent assay (ELISA). At $24 \mathrm{~h}$ post-treatment with FK506, culture media were collected and assayed for EGF secretion. The collected media were concentrated with centrifugal filter units (Merck Millipore) according to the manufacturer's protocol, and cOmplete ${ }^{\mathrm{TM}}$, Mini Protease Inhibitor Cocktail (Roche Applied Science, Penzberg, Germany) was added to samples, as previously described (18). EGF levels were assessed in triplicate using the Quantikine ${ }^{\circledR}$ RAT EGF Immunoassays (cat. no. DEG00; R\&D Systems, Inc., Minneapolis, MN, USA), according to the manufacturer's protocol. Absorbance from colorimetric reactions was determined by an ELISA reader (Biotek Instruments, Inc., Winooski, VT, USA), and normalized to protein content using a standard curve for serially diluted standard recombinant EGF.

Culture and treatment of primary neuronal cells. Spinal neurons were cultured as described previously with modifications (19). Briefly, the spinal cords of three fetal SD rats (BetterBiotechnology Co., Ltd.) were removed on embryonic day 15 (E15), and placed in dishes containing PBS. After the removal of the meninges, the spinal cords were dissected and incubated with $0.05 \%$ trypsin for $15 \mathrm{~min}$ at $37^{\circ} \mathrm{C}$. The digestion was terminated with $15 \%$ FBS DMEM/Ham's F12 (Invitrogen; Thermo Fisher Scientific, Inc.). Next, they were dissociated using a fire-polished Pasteur pipette, centrifuged at $200 \mathrm{x} \mathrm{g}$ for $5 \mathrm{~min}$ at room temperature, resuspended in DMEM/Ham's F12 containing 10\% FBS, 5\% horse serum, $100 \mathrm{U} / \mathrm{ml}$ penicillin and $100 \mu \mathrm{g} / \mathrm{ml}$ streptomycin (all Invitrogen; Thermo Fisher Scientific, Inc.), and plated on poly-L-lysine-coated $35-\mathrm{mm}$ glass bottom dish at a density of $4 \times 10^{5}$ cells $/ \mathrm{ml}$ in a humidified $5 \% \mathrm{CO}_{2}$ atmosphere at $37^{\circ} \mathrm{C}$. At $4 \mathrm{~h}$ after seeding, the glial cells were removed by washing with DMEM, and the culture medium was exchanged for the following: i) Negative control group, neurobasal medium $+2 \%$ B27 (Gibco); ii) positive control group, neurobasal medium $+2 \%$ B27 + 10 ng/ml EGF; iii) C-CM; and iv) FK506-CM or v), i.e., neutralized CM, consisting of FK506-CM and $\mathrm{C}-\mathrm{CM}$ incubated in the presence of anti-EGF neutralizing antibodies (1:100; cat. no. MAB3214; R\&D Systems) for $2 \mathrm{~h}$ at $37^{\circ} \mathrm{C}$ prior to use (20). In addition, to identify whether FK506 was able to directly promote neurite outgrowth, the total neurite length of spinal cord neurons were cultivated in neurobasal medium $+2 \% \mathrm{~B} 27+10 \mathrm{ng} / \mathrm{ml} \mathrm{EGF}$ at $37^{\circ} \mathrm{C}$ and treated with $0,10,20$ and $40 \mu \mathrm{M}$. The total neurite length was then measured following 1 and 3 days of culture.

Immunofluorescent staining. The procedure was performed as previously described (21) with minor modifications. Briefly, $24 \mathrm{~h}$ after incubation with the CM, the neuronal cells were fixed with $4 \%$ paraformaldehyde for $30 \mathrm{~min}$, washed with PBS three times, permeabilized with $0.05 \%$ Triton X-100 for 5 min, blocked with $5 \% \mathrm{BSA}$ for $30 \mathrm{~min}$ at room temperature after washing, then incubated with anti-MAP2-mouse-IgG (1:100; 
cat. no. sc-74422; Santa Cruz Biotechnology, Inc.) overnight at $4^{\circ} \mathrm{C}$. After this incubation, the samples were extensively washed with PBS and incubated with goat anti-mouse-IgG-conjugated with Alexa Fluor ${ }^{\circledR} 568$ (1:100; cat. no. A11004; Invitrogen; Thermo Fisher Scientific, Inc.) dissolved in 1\% BSA for $1 \mathrm{~h}$. Finally, all samples were stained with DAPI for 5 min, rinsed with PBS, and mounted onto microscope slides with ProLong Gold antifade reagent (Molecular Probes; Thermo Fisher Scientific, Inc.). Negative controls were performed by omitting the primary antibody during staining, and no reactivity was observed. A total of 40 fields in each group were photographed using a Laser scanning confocal microscope (Zeiss LSM710; Carl Zeiss AG) at magnification, $\mathrm{x} 20$, and images were captured with the ZEN2009 software (version 5.5 SP1; Carl Zeiss AG, Oberkochen, Germany). Fluorescent images of individual neurons were obtained.

Neurite outgrowth assay. To determine neurite outgrowth, neurite length was assessed by measuring the distance from one cell body to the end of all neurites, in which the final length was considered as the sum of all neurites measured from the cell body (22). Furthermore, the longest neurite length was measured from one cell body to the end of the longest neurite. In all groups, $\geq 80$ randomly selected neurons were observed, and only fluorescence-positive cells were scored and analyzed. The neurite length of all neurons in 10 images of each well was measured using a Zeiss LSM Image Browser software, version 4.2.0.121 (Carl Zeiss AG). The average for four wells was calculated and recorded as the mean neurite length in each condition.

Statistical analysis. Data are expressed as the mean \pm standard deviation, and the statistical analysis was performed using SPSS statistical software (version 13.0; SPSS, Inc. Chicago, IL, USA) by t-tests or one-way analysis of variance followed by the Bonferroni and Dunnett's T3 post-hoc multiple group comparison tests. The level of statistical significance is defined as $\mathrm{P}<0.05$.

\section{Results}

FK506 could improve functional recovery after SCI. As shown in Fig. 1, the mean BBB scores of the FK506-treated group and control group are 21 prior to surgery, indicating normally ambulating rodents. Mean BBB scores for all groups were recorded 2 to 3 days post-operation. On day 14 post-operation, the FK506-treated group showed significantly improved hindlimb performance compared with the control group $(\mathrm{P}<0.05)$. Furthermore, the superior recovery of FK506-treated group continued throughout the survival period; with significant improvement in BBB scores at days 35 and 42 post-operation $(\mathrm{P}<0.01)$.

FK506 had no direct promotion effect on neuronal cells, while EGF promoted neurite outgrowth. To identify whether FK506 could directly promote neurite outgrowth, the total neurite length of spinal cord neurons cultivated with $0,10,20$ and $40 \mu \mathrm{M}$ FK506 was measured for 1 or 3 days. After the cells were immunostained for MAP2, the neurite length was assessed and analyzed. FK506 treatment did not significantly

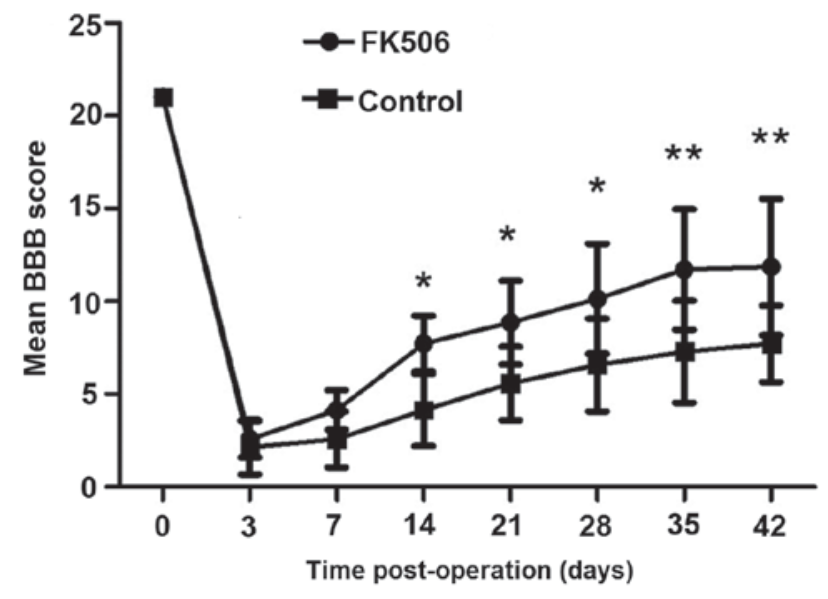

Figure 1. BBB scores recorded by open-field test. Plots showing progression of the open-field locomotor activity evaluated by BBB score over 42 days post-operation. ${ }^{*} \mathrm{P}<0.05$ and ${ }^{* *} \mathrm{P}<0.01$ vs. control group. Error bars represent the standard of the mean. BBB, Basso, Beattie and Bresnahan.

increase the total neurite length on days 1 and 3 compared with the control group in vitro (Fig. 2). The mean total neurite length of individual neurons cultured in the FK506 treatment group $(81.66 \pm 18.31 \mu \mathrm{m}$ at $10 \mu \mathrm{M}, 85.19 \pm 19.56 \mu \mathrm{m}$ at $20 \mu \mathrm{M}$, and $75.32 \pm 19.99 \mu \mathrm{m}$ at $40 \mu \mathrm{M}$ ) was similar to that of the control group (80.52 $\pm 18.30 \mu \mathrm{m} ; \mathrm{P}>0.05)$ on day 1 (Fig. $2 \mathrm{C}$ ). Furthermore, the mean total neurite length in the FK506 group was not significantly different compared with the control group on day 3 ( $\mathrm{P}>0.05$; Fig. $2 \mathrm{C}$ ). The results indicated that FK506 had no direct effect on nerve cells in promoting recovery of neurological function.

In order to verify the role of EGF to promote the growth of neurite, the total neurite length of spinal cord neurons after being cultivated for 4 days with $10 \mathrm{ng} / \mathrm{ml}$ EGF was measured. The results showed that the total neurite length of individual neurons cultured with EGF was markedly longer compared with the control group (Fig. 3).

FK506-CM could increase neurite outgrowth. To investigate the role of astrocytes as mediators of the neuroprotective effects of FK506, the total and longest neurite length of spinal cord neurons cultivated with various CM for 1 and 3 days were measured. After the cells were immunostained for MAP2, the neurite length was assessed and analyzed. The results indicated that treatment with FK506-CM induced a $61.06 \%$ increase in total neurite length on day 1 (Fig. 4), and $56.4 \%$ on day 3 compared with the C-CM group (Fig. 5). After incubation with CMs for one day, the mean length of the total neurite of individual neurons cultured in FK506-CM was $181.79 \pm 23.73 \mu \mathrm{m}$, which was significantly longer than those in the $\mathrm{C}-\mathrm{CM}$ group $(112.88 \pm 20.48 \mu \mathrm{m} ; \mathrm{P}<0.01)$ and those in the control group $(72.68 \pm 18.57 \mu \mathrm{m} ; \mathrm{P}<0.01$; Fig. $4 \mathrm{~F})$. By contrast, the mean lengths of total neurites on day 3 were $270.77 \pm 48.67,173.13 \pm 27.68$ and $122.74 \pm 27.84 \mu \mathrm{m}$ in the FK506-CM, C-CM and control groups, respectively $(\mathrm{P}<0.01$; Fig. 5F). Similar results were observed when only the longest neurite was measured (Figs. 4G and 5G). Thus, analysis of neuronal morphology revealed a marked increase in the 

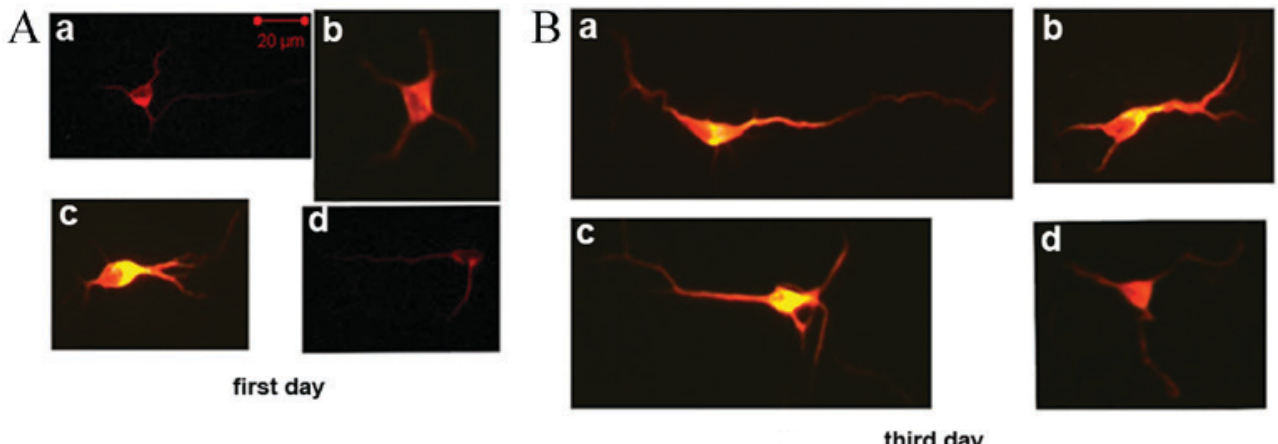

third day

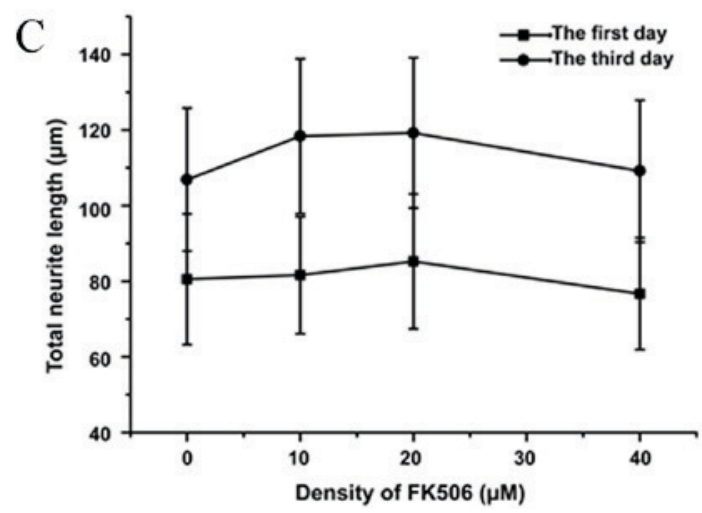

Figure 2. FK506 did not promote neurite outgrowth directly on days 1 and 3. (A) Micrographs of rat neurons cultured in control medium and FK506 at various doses on day 1. (a) Control, (b) FK506 at $10 \mu \mathrm{M}$, (c) FK506 at $20 \mu \mathrm{M}$ and (d) FK506 at $40 \mu \mathrm{M}$. (B) Micrographs of rat neurons cultured in control medium and FK506 with different doses on day 3. (a) Control, (b) FK506 at $10 \mu \mathrm{M}$, (c) FK506 at $20 \mu \mathrm{M}$ and (d) FK506 at $40 \mu \mathrm{M}$. (C) Quantification of total neurite length following treatment with FK506 on days 1 and 3. Neurons were stained with microtubule associated protein 2 antibody (red). Results are expressed as the mean \pm standard deviation. A minimum of 80 neurons per condition were analyzed. Statistical comparisons between means were made with one-way analysis of variance.

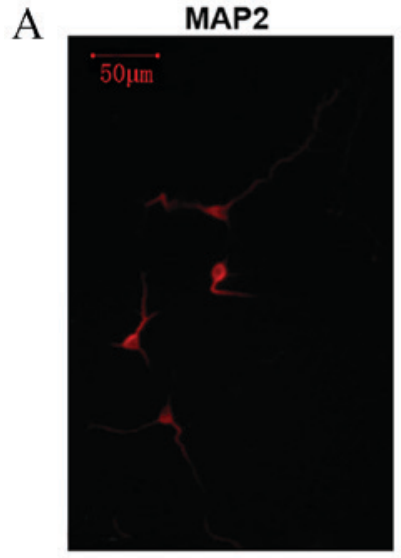

MAP2
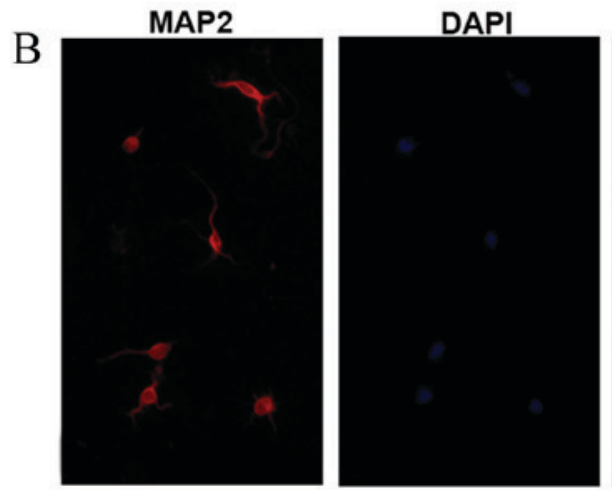

Merge

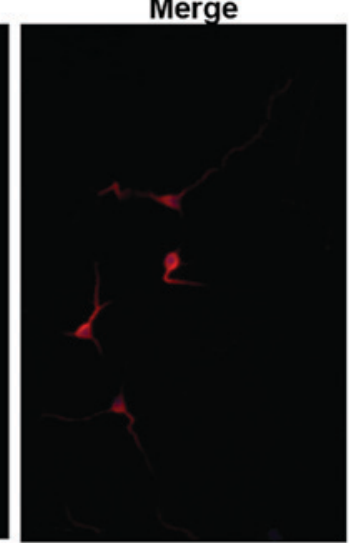

Merge

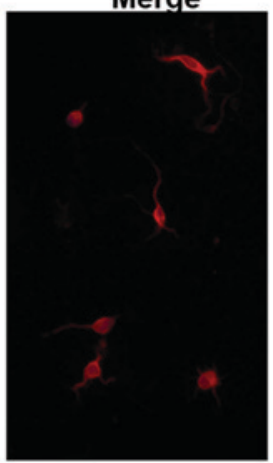

Figure 3. EGF can promote neurite outgrowth. Representative micrographs of (A) EGF-treated and (B) control rat neurons at 4 days. As shown above, neurons were stained with MAP2 antibody (red) and DAPI for nuclei (blue). Total neurite length of spinal cord neurons cultured with EGF were markedly longer than the control group. EGF, epidermal growth factor; MAP2, microtubule associated protein 2. 

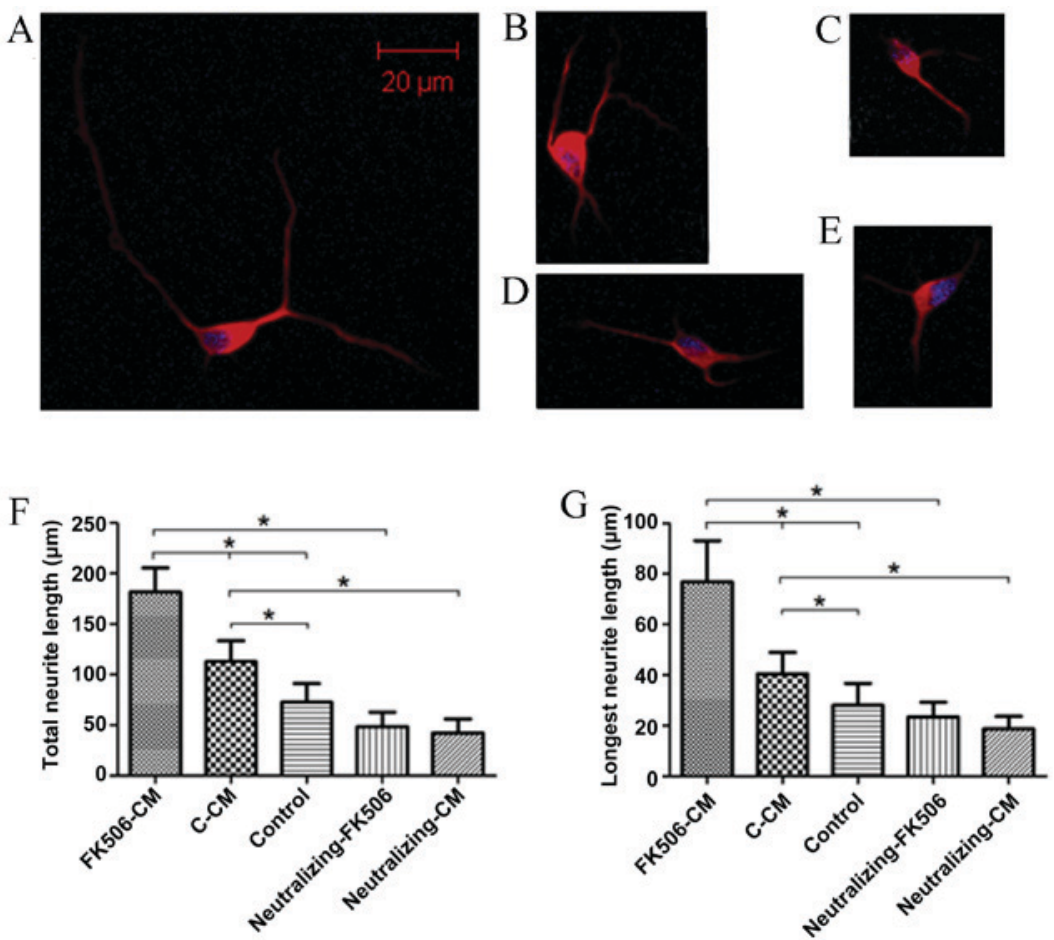

Figure 4. FK506 was found to enhance neurite outgrowth on day 1 after treatments with CM. Representative micrographs of rat neurons cultured in (A) FK506-CM, (B) C-CM, (C) control, (D) neutralizing-C-CM or (E) neutralizing-FK506-CM for $24 \mathrm{~h}$. (F) Quantification of total neurite length and (G) longest neurite length of spinal cord neurons. FK506-CM and C-CM were able to significantly promote neurite growth compared with the control group, of which FK506-CM group exhibited the most obvious effect. The neutralizing-FK506-CM and neutralizing-C-CM groups noticeably inhibited neurite growth The results indicated that FK506 was dependent on EGF stimulation in order to affect neurite outgrowth. Neurons were stained with MAP2 antibody (red) and DAPI for nuclei (blue). Results are expressed as the mean \pm standard deviation. A minimum of 80 neurons in each of the CMs were analyzed. Statistical comparisons between means were made with one-way analysis of variance ( $\mathrm{P}<0.01)$. CM, conditioned media; $\mathrm{C}-\mathrm{CM}$, conditioned media of the control group.
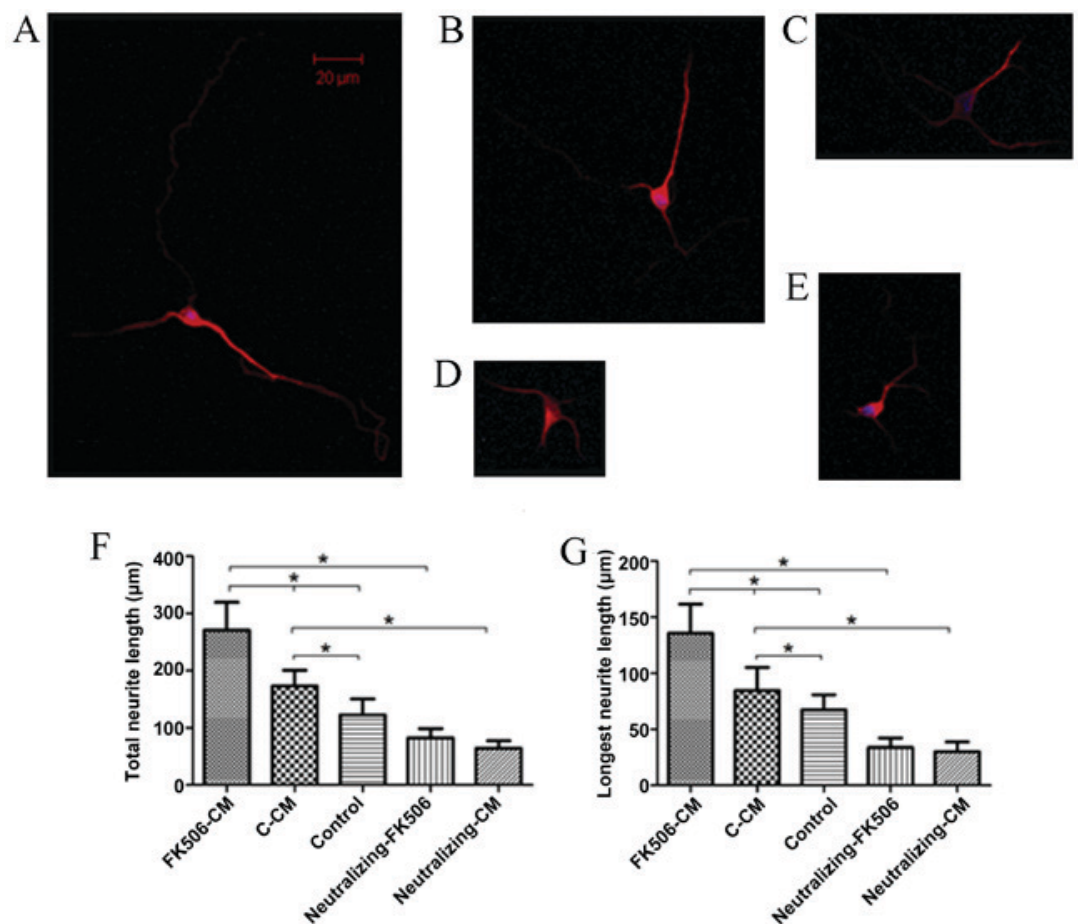

Figure 5. FK506 was found to enhance neurite outgrowth on the third day after treatment with CM. Representative micrographs of rat neurons cultured in (A), FK506-CM, (B) C-CM, (C) control, (E) neutralizing-FK506-CM or (D) neutralizing-C-CM for 3 days. (F) Quantification of total neurite length and (G) longest neurite length of spinal cord neurons. Both FK506-CM and C-CM were able to significantly promote neurite growth compared with the control group, in which the effects of treatment with FK506-CM were most pronounced. Both the neutralizing-FK506-CM and neutralizing-C-CM groups displayed noticeable inhibition of neurite growth. The results indicated that FK506 was dependent on EGF stimulation in order to affect neurite outgrowth. Neurons were stained with MAP2 antibody (red) and DAPI for nuclei (blue). Results are expressed as the mean \pm standard deviation. A minimum of 80 neurons in each of the CMs were analyzed. Statistical comparisons between means were made with one-way analysis of variance ("P<0.01). EGF, epidermal growth factor; MAP2, microtubule associated protein 2. 


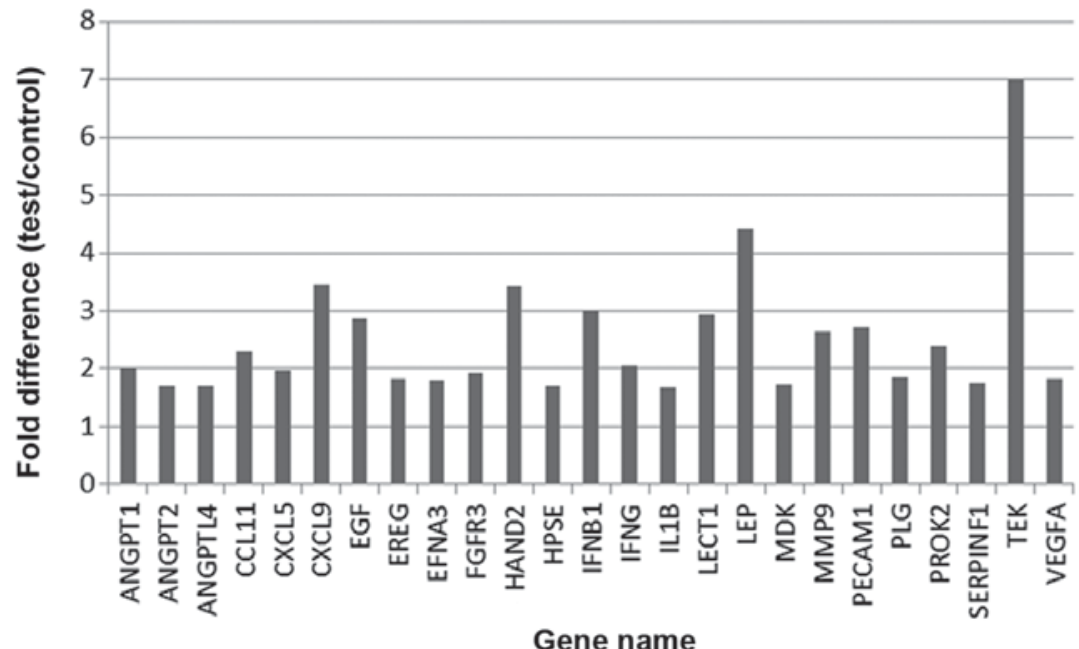

Figure 6. Microarray results depicting the significantly elevated levels of cytokines subsequent to treatment with FK506. A total of 25 associated genes were upregulated >1.6-fold after FK506 treatment, wherein epidermal growth factor levels increased markedly.
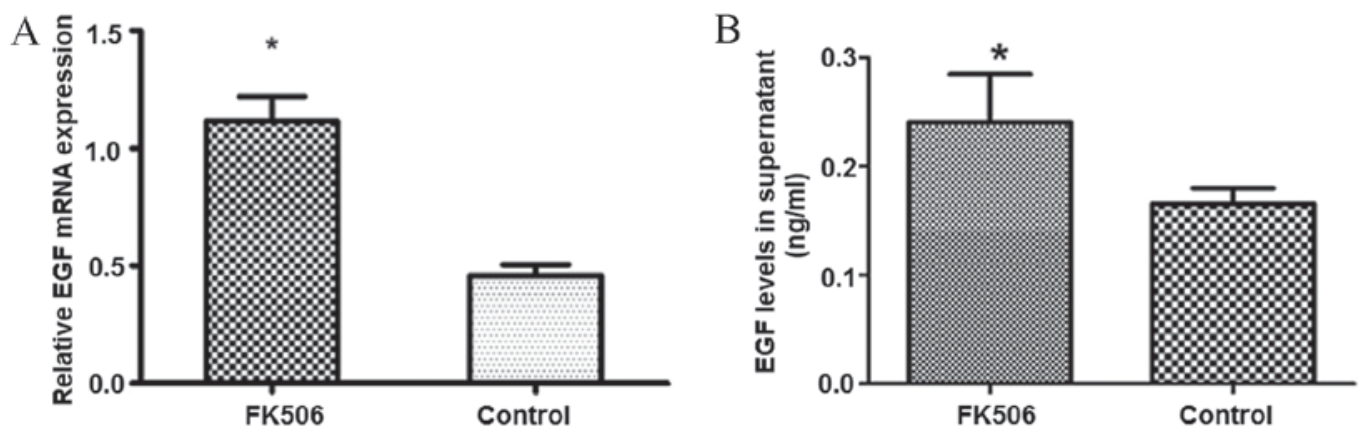

Figure 7. Expression levels of EGF mRNA in astrocytes and EGF protein expression levels in the supernatant. (A) EGF mRNA expression levels were significantly increased in astrocytes treated with FK506 for $8 \mathrm{~h}$ compared with the control. (B) The EGF concentration levels in the supernatant were measured using ELISA. The concentration levels of EGF in FK506-CM was significantly higher compared with the control. "P<0.05 vs. control. RT-qPCR, reverse transcription-quantitative polymerase chain reaction; EGF, epidermal growth factor.

neurite outgrowth when the spinal neurons were treated with FK506-CM.

FK506 stimulated astrocyte expression of EGF in vitro and in vivo. In the present study, gene chip detection of astrocytes treated with FK506 was performed. The results showed that a total of 25 significantly upregulated genes were identified in the astrocytes treated with FK506 $(\mathrm{P}<0.05)$. Furthermore, EGF displayed elevated levels of the cytokines, as shown in Fig. 6.

To further examine the effect of FK506 on the expression levels of EGF, which is produced by astrocytes, total RNA was extracted from the monolayers of astrocytes, and mRNA expression levels of EGF were quantitatively evaluated by RT-qPCR, and EGF protein expression levels were measured by the ELISA method. The results indicated that the RNA expression levels of EGF in astrocytes treated with FK506 were 2.4-times higher compared with those of the control group, and EGF protein levels in the supernatant were also significantly increased compared with the control group ( $\mathrm{P}<0.05$; Fig. 7).

In addition, the present study examined the effect of FK506 on the ability of astrocytes to produce EGF in vivo (Fig. 8). Following a contusion of the spinal cord, rats were randomly and blindly assigned to the FK506 $(0.5 \mathrm{mg} / \mathrm{kg})$ or vehicle treatment groups. Immunohistochemical analysis of EGF and GFAP double staining was performed. The results indicated that sections from FK506-treated groups showed strongly EGF-immunoreactive astrocytes. These markedly EGF-immunoreactive astrocytes were predominantly in the vicinity of the lesion $24 \mathrm{~h}$ post-injury, reaching peak levels in the initial 3 days, and gradually decreasing until day 14 (Fig. 8A, C, E and G). By contrast, the EGF expression levels in the control group were markedly lower compared with those in the FK506-treated group (Fig. 8B, D, F and H). Additionally, the present study found that the level of EGF-immunoreactive astrocytes in the control group was perceptibly decreased on the third day compared with those detected at $24 \mathrm{~h}$. After 7 days, few EGF-positive astrocytes remained in the control group.

FK506-CM-induced promotion of neuritogenesis may be interrupted by EGF neutralizing antibodies. To further verify the involvement of astrocyte-derived EGF on neurite outgrowth, embryonic spinal neurons were cultured in neutralized FK506-CM and neutralized C-CM, which had been pre-treated with EGF neutralizing antibodies, and subsequently the length of neurites were analyzed (Figs. 4 and 5). 


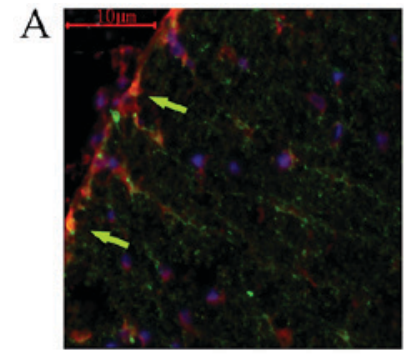

E

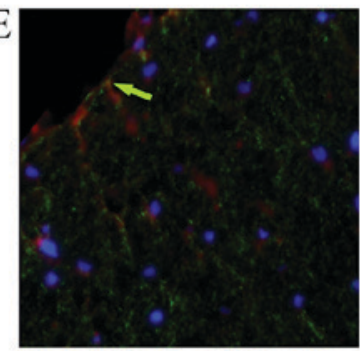

B

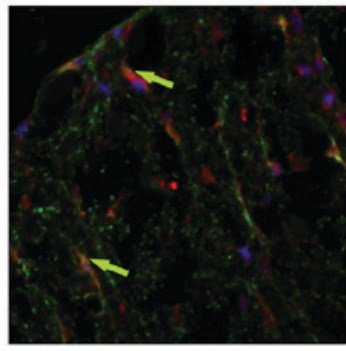

$\mathrm{F}$

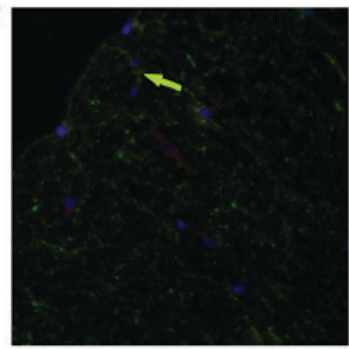

C

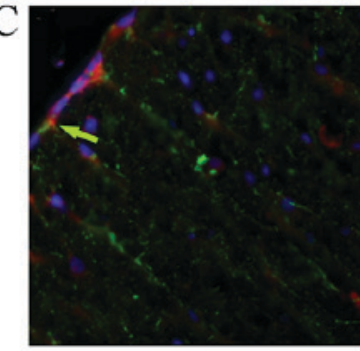

$\mathrm{G}$

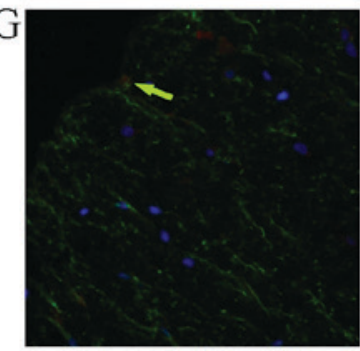

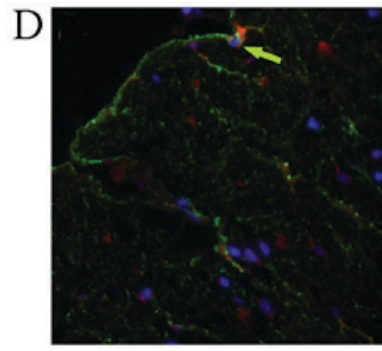

$\mathrm{H}$

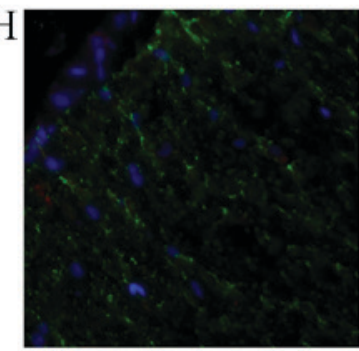

Figure 8. FK506 modulated astrocyte production of EGF in vivo. The expression levels of EGF in astrocytes was markedly increased in rats treated with FK506 on days 1, 3 and 7, compared with those in the normal control. However, EGF expression was decreased markedly after day 7 post-surgery. FK506 treated groups: (A) day 1; (B) day 3; (C) day 7; (D) day 14; and control groups: (E), day 1; (F), day 3; (G), day 7; and (H) day 14. Red indicates immunolabeling for EGF, green for GFAP, blue for DAPI, and yellow for the double staining with EGF and GFAP. EGF, epidermal growth factor; GFAP, glial fibrillary acidic protein.

The results demonstrated that the increase in neurite length was reduced following treatment with neutralized $\mathrm{C}-\mathrm{CM}$ and neutralized FK506-CM, compared with the non-neutralized C-CM and FK506-CM, on days 1 and 3. The total length of neurites was reduced by 25.71 and $37.46 \%$ in the neutralized FK506-CM and neutralized C-CM groups, respectively on day 1 compared with the control. For day 3 these values were 30.52 and $36.16 \%$, respectively. The same results were found with respect to the longest neurite length. Furthermore, to exclude non-specific inhibitory effects with the solution or preservatives involved in the antibody preparation, control experiments were performed, which showed that none of these agents altered neurite elongation (data not shown). Thus, the results indicated that astrocytic EGF secretion in response to FK506 treatment serves a significant role in neurite elongation.

\section{Discussion}

In the present study it was found that that FK506 was able to enhance neurite outgrowth and improve the functional recovery of the spinal cord by stimulating astrocytes to secrete EGF. This is an indirect effect of FK506 on neuronal cells by an astrocyte-mediated process.

Numerous studies support the hypothesis that FK506 is able to improve functional recovery and nerve regeneration after nerve injury $(23,24)$. For instance, López-Vales et al $(25)$ previously demonstrated that the administration of FK506 30 min after SCI was effective in inducing neuroprotection and functional preservation. In accordance with previous studies, the present results showed that FK506 could improve locomotor functional recovery of the limbs of rats after SCI, as quantified by the BBB score. Furthermore, the study by López-Vales et al showed that repeated treatment with FK506 could improve histologic and functional outcomes to a greater degree than a single administration of FK506. In addition, a bell-shaped dose-response curve of FK506 on the rate of axonal regeneration and on neurite outgrowth has been reported $(26,27)$. The current study indicated little promotive effect of FK506 on neurite outgrowth in a human neuronal cell line in vitro with different doses $(10,20$ and $40 \mu \mathrm{M})$ for 1 and 3 days, which suggested that FK506 may have an indirect effect on neurite outgrowth; however, further investigations are warranted.

Reactive astrogliosis is initiated when trigger factors produced at the site of injury drive astrocytes to become activated from their quiescent state (28). The reactive astrocytes are reported to have a dual role with respect to their overall beneficial or detrimental effect on neuroprotection, tissue regeneration and functional recovery (28). A study by Bush et al (29) demonstrated that ablation of proliferating astrocytes after SCI could lead to increased neuronal degeneration and motor deficits. Therefore, astrocytes responses at the site of injury may contribute to neuroprotection and functional recovery after SCI. Additionally, Szydlowska et al (30) demonstrated that FK506 could block the activation of extracellular signal-regulated kinases 1 and 2 signaling in glutamate-induced death of astrocytes and astrocytic cell death in vitro and in ischemic brains. We hypothesized that FK506 could provide a neuroprotective effect by modulating the activity of astrocytes. Szydlowska et al (31) reported that FK506 may inhibit glutamate-induced astrocyte death. In addition, a previous study demonstrated that FK506 could substantially reduce the rise of the effective concentration of the $\mathrm{Ca}^{2+}$ ionophore in astrocytes, which is likely to be responsible for their protection against mitochondrial depolarization and cell death (32). In the present study, spinal neuronal cells were cultured with FK506-CM, which was the supernatant of FK506-treated astrocyte culture, and a marked increase in neurite length was observed. Thus, the results suggested that FK506 may stimulate astrocytes to secrete certain cytokines, which could significantly enhance neurite outgrowth.

Furthermore, the results of RT-qPCR and EGF-neutralizing assessments demonstrated that the level of EGF in the FK506-CM group is significantly higher than that of the 
control group. EGF is a highly mitogenic factor in numerous mammalian cell types (33), and is able to promote the proliferation and differentiation of neuronal progenitors, postmitotic neurons and glial cells in the central nervous system $(34,35)$. EGF is also an important neurotrophic factor and can stimulate neurite outgrowth in a previous study (36). In addition, studies have reported that EGF can modulate neurite extension by stimulating thyroid hormones and versican G3 domain $(37,38)$. Modulation of the expression of glial-derived neurotrophic factor has been considered as a potential neuroprotective mechanism of immunophilin ligands (39). Furthermore, the results in the current study showed that astrocytes could be stimulated to secrete EGF by treatment with FK506, which is a potent neurotrophic factor and could enhance neurite outgrowth in neuronal cell lines. In addition, the neuroprotective action of EGF in the FK506-CM group is interrupted by EGF neutralizing antibodies. Thus, we suggest that the astrocytic EGF secretion in response to FK506 treatment serves an important role in the neuroprotective effect of FK506.

In conclusion, the present study demonstrated that FK506 has neuroprotective activity in repairing SCI by stimulating astrocytes to secrete EGF. However, the current study only reported the effects of the administration of FK506 within $30 \mathrm{~min}$ post-SCI at a single dose. Further experiments and studies are required to examine the consequence of repeated treatment with different doses of FK506. Furthermore, the present study was not able to elucidate the concrete mechanism underlying EGF-induced neurite outgrowth, and further efforts are warranted to clarify this mechanism in future investigations. Thus, the present study may provide insights into the complexity of cell-cell interactions during SCI, and also demonstrated a potential effective treatment strategy for SCI, based on the promotion of neural repair and functional recovery.

\section{Acknowledgements}

The present study was supported by the National Natural Science Foundation of China (grant no. 81171694).

\section{References}

1. Ibarra A and Martiñón S: Pharmacological approaches to induce neuroregeneration in spinal cord injury: An overview. Curr Drug Discov Technol 6: 82-90, 2009.

2. Baptiste DC and Fehlings MG: Update on the treatment of spinal cord injury. Prog Brain Res 161: 217-233, 2007.

3. Faulkner JR, Herrmann JE, Woo MJ, Tansey KE, Doan NB and Sofroniew MV: Reactive astrocytes protect tissue and preserve function after spinal cord injury. J Neurosci 24: 2143-2155, 2004.

4. Davies S, Shih CH, Noble M, Mayer-Proschel M, Davies JE and Proschel C: Transplantation of specific human astrocytes promotes functional recovery after spinal cord injury. PLoS One 6: e17328, 2011.

5. Staatz CE and Tett SE: Clinical pharmacokinetics and pharmacodynamics of tacrolimus in solid organ transplantation. Clin Pharmacokinet 43: 623-653, 2004.

6. Klettner A and Herdegen T: FK506 and its analogs-therapeutic potential for neurological disorders. Curr Drug Targets CNS Neurol Disord 2: 153-162, 2003.

7. Sosa I, Reyes O and Kuffler D: Immunosuppressants: Neuroprotection and promoting neurological recovery following peripheral nerve and spinal cord lesions. Exp Neurol 195: 7-15, 2005.

8. Wildering WC, Hermann PM and Bulloch AG: Lymnaea epidermal growth factor promotes axonal regeneration in CNS organ culture. J Neurosci 21: 9345-9354, 2001.
9. Liberto CM, Albrecht PJ, Herx LM, Yong VW and Levison SW: Pro-regenerative properties of cytokine-activated astrocytes. J Neurochem 89: 1092-1100, 2004

10. Kuh SU, Cho YE, Yoon DH, Kim KN and Ha Y: Functional recovery after human umbilical cord blood cells transplantation with brain-derived neutrophic factor into the spinal cord injured rat. Acta Neurochir (Wien) 147: 985-992, 2005.

11. Young W: Spinal cord regeneration. Science 273: 451, 1996.

12. Basso DM, Beattie MS and Bresnahan JC: A sensitive and reliable locomotor rating scale for open field testing in rats. J Neurotrauma 12: 1-21, 1995.

13. Zhang A, Zhang J, Sun P, Yao C, Su C, Sui T, Huang H, Cao X and Ge Y: EIF2alpha and caspase-12 activation are involved in oxygen-glucose-serum deprivation/restoration-induced apoptosis of spinal cord astrocytes. Neurosci Lett 478: 32-36, 2010.

14. Iyer VR, Eisen MB, Ross DT, Schuler G, Moore T, Lee JC, Trent JM, Staudt LM, Hudson J Jr, Boguski MS, et al: The transcriptional program in the response of human fibroblasts to serum. Science 283: 83-87, 1999

15. Livak KJ and Schmittgen TD: Analysis of relative gene expression data using real-time quantitative PCR and the 2(-Delta Delta C(T)) method. Methods 25: 402-408, 2001.

16. Liu N, Li H, Liu K, Yu J, Cheng M, De W, Liu J, Shi S, He Y and Zhao J: Differential expression of genes and proteins associated with wool follicle cycling. Mol Biol Rep 41: 5343-5349, 2014.

17. Cai J, Zhao XL, Liu AW, Nian H and Zhang SH: Apigenin inhibits hepatoma cell growth through alteration of gene expression patterns. Phytomedicine 18: 366-373, 2011.

18. Gezginci-Oktayoglu S, Karatug A and Bolkent S: The relation among NGF, EGF and insulin is important for triggering pancreatic $\beta$ cell apoptosis. Diabetes Metab Res Rev 28: 654-662, 2012.

19. Han SS, Kang DY, Mujtaba T, Rao MS and Fischer I: Grafted lineage-restricted precursors differentiate exclusively into neurons in the adult spinal cord. Exp Neurol 177: 360-375, 2002.

20. Gomes FC, Maia CG, de Menezes JR and Neto VM: Cerebellar astrocytes treated by thyroid hormone modulate neuronal proliferation. Glia 25: 247-255, 1999.

21. Stipursky J and Gomes FC: TGF-beta1/SMAD signaling induces astrocyte fate commitment in vitro: Implications for radial glia development. Glia 55: 1023-1033, 2007.

22. E Spohr TC, Dezonne RS, Rehen SK and Gomes FC: Astrocytes treated by lysophosphatidic acid induce axonal outgrowth of cortical progenitors through extracellular matrix protein and epidermal growth factor signaling pathway. J Neurochem 119: 113-123, 2011.

23. Voda J, Yamaji T and Gold BG: Neuroimmunophilin ligands improve functional recovery and increase axonal growth after spinal cord hemisection in rats. J Neurotrauma 22: 1150-1161, 2005.

24. Yeh C, Bowers D and Hadlock TA: Effect of FK506 on functional recovery after facial nerve injury in the rat. Arch Facial Plast Surg 9: 333-339, 2007.

25. López-Vales R, García-Alías G, Forés J, Udina E, Gold BG, Navarro X and Verdú E: FK506 reduces tissue damage and prevents functional deficit after spinal cord injury in the rat. J Neurosci Res 81: 827-836, 2005.

26. Udina E, Ceballos D, Verdú E, Gold BG and Navarro X: Bimodal dose-dependence of FK506 on the rate of axonal regeneration in mouse peripheral nerve. Muscle Nerve 26: 348-355, 2002.

27. Gold BG, Densmore V, Shou W, Matzuk MM and Gordon HS: Immunophilin FK506-binding protein 52 (not FK506-binding protein 12) mediates the neurotrophic action of FK506. J Pharmacol Exp Ther 289: 1202-1210, 1999.

28. Buffo A, Rolando C and Ceruti S: Astrocytes in the damaged brain: Molecular and cellular insights into their reactive response and healing potential. Biochem Pharmacol 79: 77-89, 2010.

29. Bush TG, Puvanachandra N, Horner CH, Polito A, Ostenfeld T, Svendsen CN, Mucke L, Johnson MH and Sofroniew MV: Leukocyte infiltration, neuronal degeneration, and neurite outgrowth after ablation of scar-forming, reactive astrocytes in adult transgenic mice. Neuron 23: 297-308, 1999.

30. Szydlowska K, Gozdz A, Dabrowski M, Zawadzka M and Kaminska B: Prolonged activation of ERK triggers glutamate-induced apoptosis of astrocytes: Neuroprotective effect of FK506. J Neurochem 113: 904-918, 2010.

31. Szydlowska K, Zawadzka M and Kaminska B: Neuroprotectant FK506 inhibits glutamate-induced apoptosis of astrocytes in vitro and in vivo. J Neurochem 99: 965-975, 2006. 
32. Kahraman S, Bambrick LL and Fiskum G: Effects of FK506 and cyclosporin a on calcium ionophore-induced mitochondrial depolarization and cytosolic calcium in astrocytes and neurons. J Neurosci Res 89: 1973-1978, 2011.

33. Wong RW and Guillaud L: The role of epidermal growth factor and its receptors in mammalian CNS. Cytokine Growth Factor Rev 15: 147-156, 2004.

34. Fricker-Gates RA, Winkler C, Kirik D, Rosenblad C, Carpenter MK and Bjorklund A: EGF infusion stimulates the proliferation and migration of embryonic progenitor cells transplanted in the adult rat striatum. Exp Neurol 165: 237-247, 2000.

35. Yamada $\mathrm{M}$, Ikeuchi $\mathrm{T}$ and Hatanaka $\mathrm{H}$ : The neurotrophic action and signalling of epidermal growth factor. Prog Neurobiol 51: 19-37, 1997.

36. Goldshmit Y, Greenhalgh CJ and Turnley AM: Suppressor of cytokine signalling-2 and epidermal growth factor regulate neurite outgrowth of cortical neurons. Eur J Neurosci 20: 2260-2266, 2004.
37. Martinez R and Gomes FC: Neuritogenesis induced by thyroid hormone-treated astrocytes is mediated by epidermal growth factor/mitogen-activated protein kinase-phosphatidylinositol 3-kinase pathways and involves modulation of extracellular matrix proteins. J Biol Chem 277: 49311-49318, 2002.

38. Xiang YY, Dong H, Wan Y, Li J, Yee A, Yang BB and Lu WY: Versican G3 domain regulates neurite growth and synaptic transmission of hippocampal neurons by activation of epidermal growth factor receptor. J Biol Chem 281: 19358-19368, 2006.

39. Zawadzka M and Kaminska B: Immunosuppressant FK506 affects multiple signaling pathways and modulates gene expression in astrocytes. Mol Cell Neurosci 22: 202-209, 2003.

(i) (9) This work is licensed under a Creative Commons Attribution-NonCommercial-NoDerivatives 4.0 International (CC BY-NC-ND 4.0) License. 